\title{
Measurement of Gamma-Rays Using Smartphones
}

\author{
Sandy Tith, Nares Chankow* \\ Department of Nuclear Engineering, Faculty of Engineering, Chulalongkorn University, Bangkok, Thailand \\ Email: sandytith@gmail.com, nares.c@chula.ac.th
}

Received 2 December 2015; accepted 24 January 2016; published 27 January 2016

Copyright (C) 2016 by authors and Scientific Research Publishing Inc.

This work is licensed under the Creative Commons Attribution International License (CC BY). http://creativecommons.org/licenses/by/4.0/

(c) (;) Open Access

\section{Abstract}

The CMOS (Complementary Metal Oxide Semiconductor) image sensor of a smartphone has been known for its sensitivity to gamma-rays. In this research, some smartphones were selected and tested for measurement of gamma-rays emitted from Cesium-137 and Iridium-192 sources. During measurements, the phones were set in video mode while the camera lenses were covered with black adhesive tape to prevent light exposure. Interaction of gamma-rays with the CMOS appeared as flashing bright spots on the image. The bright spots were then counted by using the freely available ImageJ software. Preliminary results indicated that the number of bright spots increased linearly with increase of gamma-ray dose rate. An in-house Android application software was then developed for real-time counting of the bright spots. The application software also allowed users to input a calibration equation so that the phones could simultaneously convert the count rate to display in dose rate. This research demonstrated that, after appropriate calibration, smartphones could be used as gamma-ray measuring devices for radiation safety control involving high activity sources such as in industrial radiography, gamma-ray irradiation facility and medical treatment.

\section{Keywords}

Gamma-Ray, Dose Rate, Smartphones, Radiation Safety, Industrial Radiography, CMOS Image Sensor, Cesium-137, Iridium-192, Android

\section{Introduction}

Gamma rays have been extensively used in medicine, industry, agriculture, education and research. Gamma-rays are electromagnetic radiation like visible light but they have energy approximately $10^{4}$ to $10^{6}$ times higher than that of the visible light. They can penetrate matter making them very useful in various ways. In medicine, gam-

"Corresponding author. 
ma-rays play important roles in diagnosis and therapy. Various gamma-ray applications in industry have been proven and widely used, for example radiography, thickness measurement, level measurement, sterilization of products, etc. Radiation workers are trained to protect themselves and the public from receiving radiation dose beyond the allowable limits. The whole body annual dose limit for radiation workers is 50 millisievert (mSv) or 5 rem but must not exceed the average of $20 \mathrm{mSv}$ per year during any 5 consecutive years. For better radiation safety control, the dose rate of 10 microsievert per hour ( $\mu \mathrm{SV} / \mathrm{hr}$ ) is limited so that working totally 2000 hours per year (8 hours a day, 5 days a week for 50 weeks) will not exceed the annual dose limit of $20 \mathrm{mSv}$. Radiation workers must wear personal dosimeter like film badges which are completely replaced by thermoluminescent dosimeters (TLD's) and more recently optically stimulated luminescent dosimeters (OSL's) for measurement of cumulative dose from 1 month up to 3 months. In normal practice, however, dosimeters for short term measurement are also required such as digital personal dosimeter and survey meters which are not available for all workers.

It has been known and tested that the CMOS (Complementary Metal Oxide Semiconductor) image sensor chip in digital camera is sensitive to gamma and X-rays [1]-[4]. All modern smartphones come with high quality digital cameras which may be used for measurement of gamma-rays. In this research, four Android smartphones are selected according to their CMOS sensitive areas, number of image pixels, cost and availability, to test their responses to gamma-rays at different energies and dose rates. Application software is also developed so that the smartphones can display the counting rate and the dose rate simultaneously.

\subsection{Materials}

Four smartphones including Samsung Galaxy GT-S5570 mini, Samsung Galaxy SII, Samsung Galaxy SIII and Huawei Ascend P7, are first selected. Their specifications are shown in Table 1 below. They are tested in measurement of gamma-rays from the commonly used gamma-ray sources including cobalt- $60\left({ }^{60} \mathrm{Co}\right)$, Cesium-137 $\left({ }^{137} \mathrm{Cs}\right)$ and Iridium-192 ( $\left.{ }^{192} \mathrm{Ir}\right)$ with known activities to compare their sensitivities and to investigate relationship between the dose rate and the counting rate. The characteristics of the gamma-ray sources are showed in Table 2 .

\subsection{Methodology}

The selected smartphones were first exposed to gamma-rays from an ${ }^{192}$ Ir industrial radiography source at vari-

Table 1. Specifications of the selected smartphones.

\begin{tabular}{|c|c|c|c|c|}
\hline Smartphones (back camera) & $\begin{array}{l}\text { Max. image pixels } \\
\text { (photo mode) }\end{array}$ & Frame rate & $\begin{array}{l}\text { Max. image pixels } \\
\text { (video mode) }\end{array}$ & Aperture \\
\hline Samsung Galaxy GT-S5570 mini & $3.15 \mathrm{MP}$ & $14 \mathrm{fps}$ & $320 \times 240$ & No data \\
\hline Samsung Galaxy SII & $8 \mathrm{MP}$ & $24 \mathrm{fps}$ & $1920 \times 1080$ & $\mathrm{f} / 2.7$ \\
\hline Samsung Galaxy SIII & $8 \mathrm{MP}$ & $30 \mathrm{fps}$ & $1920 \times 1080$ & $\mathrm{f} / 2.6$ \\
\hline Huawei Ascend P7 & $13 \mathrm{MP}$ & $30 \mathrm{fps}$ & $1920 \times 1080$ & $\mathrm{f} / 2.0$ \\
\hline
\end{tabular}

Table 2. Gamma-ray sources and their prominent gamma-rays [5] [6].

\begin{tabular}{|c|c|c|c|c|}
\hline Gamma-ray sources & Half-life & Gamma-ray energy (keV) & Emission probability & $\Gamma^{*}(\mathrm{mSv} / \mathrm{hr}-\mathrm{Ci}$ at $1 \mathrm{~m})$ \\
\hline${ }^{137} \mathrm{Cs}$ & 30 years & 662 & $85 \%$ & 33 \\
\hline${ }^{192} \mathrm{Ir}$ & 74.2 days & $\begin{array}{l}296 \\
308 \\
317 \\
468 \\
589 \\
604 \\
612\end{array}$ & $\begin{array}{c}29 \% \\
30 \% \\
81 \% \\
49 \% \\
4 \% \\
9 \% \\
6 \%\end{array}$ & 5 \\
\hline${ }^{60} \mathrm{Co}$ & 5.26 years & $\begin{array}{l}1173 \\
1332\end{array}$ & $\begin{array}{l}100 \% \\
100 \%\end{array}$ & 133 \\
\hline
\end{tabular}

\footnotetext{
*Specific gamma-ray constant is the dose rate at $1 \mathrm{~m}$ distance from 1 curie (Ci) source.
} 
ous distances to obtain the dose rates up to about $240 \mathrm{mSv} / \mathrm{hr}$. The measurements were carried out in video mode with black adhesive tape on the camera lenses making the image background dark. Interactions of gammarays with the CMOS chips appear as flashing bright spots on dark background which could be clearly observed as showed in Figure 1. After measurement, the video file was transferred to a personal computer then converted to pictures by using the freely downloadable AOAO Video to Picture Converter software [7]. The numbers of bright spots in all pictures were finally counted using the Image J software [8] which could also be freely available. The total number of count was divided by the counting time to express in count rate. Importantly, from our investigation one interaction of gamma photon could cause the bright spots size up to 10 pixels depending on type of CMOS chip. Thus a cluster of bright spots from 1 to 10 pixels were counted as 1 count. The calibration procedure is summarized in Figure 2. It was found that the Samsung Galaxy GT-S5570 mini had lowest sensitivity among the four smartphones while Huawei Ascend P7 had the highest sensitivity as illustrated graphically in Figure 3. After that smartphones were tested with gamma-rays from ${ }^{137} \mathrm{Cs}$ and ${ }^{60} \mathrm{Co}$ sources at various dose rates in order to test their sensitivities to high energy gamma-rays. The results are illustrated graphically in Figures 4-6. The sensitivity to high energy gamma-rays was found to be less than to ${ }^{192}$ Ir due to lower interaction probability as predicted. Application software was finally developed for the Android smartphones so that they could be used to measure gamma-rays and display the results in both counting rate and the dose rate on the
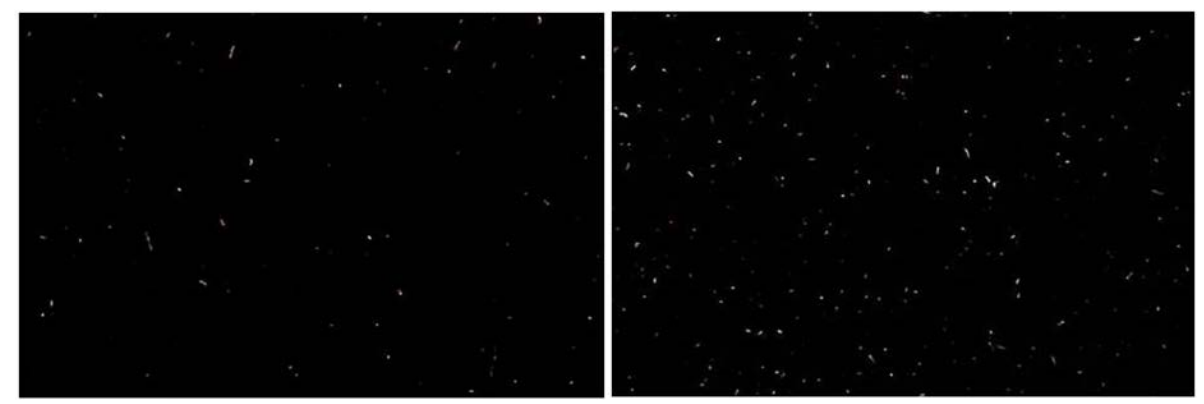

Figure 1. Showing bright spots on dark background image caused by interaction of gammarays with digital camera's CMOS chip at low (left) and high (right) gamma dose rates.

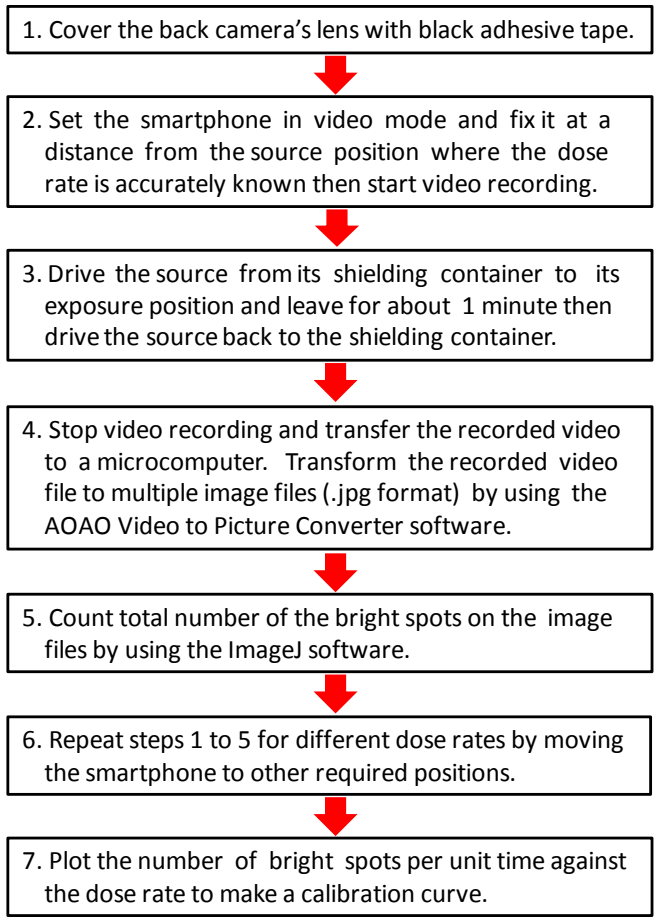

Figure 2. Calibration procedure. 


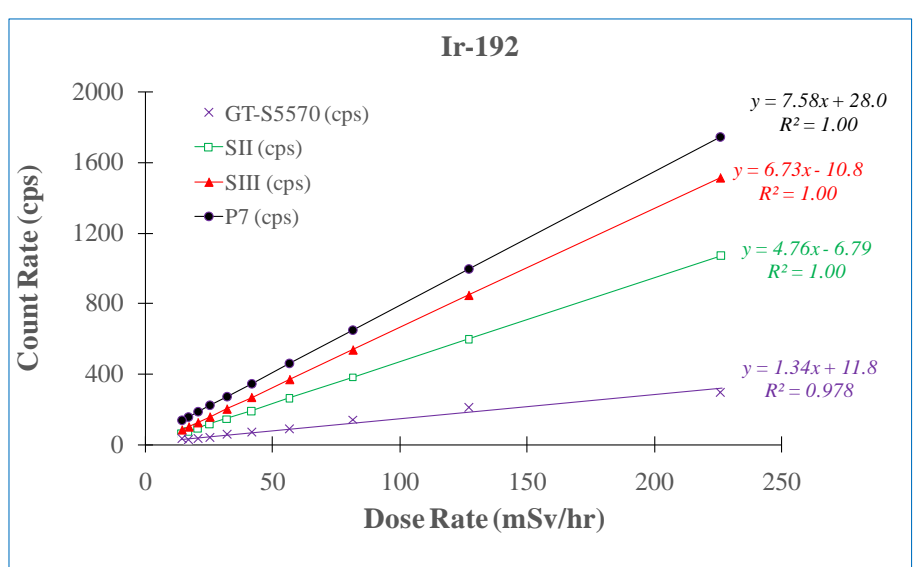

Figure 3. Gamma count rates vs the dose rates from Ir-192 industrial radiography source.

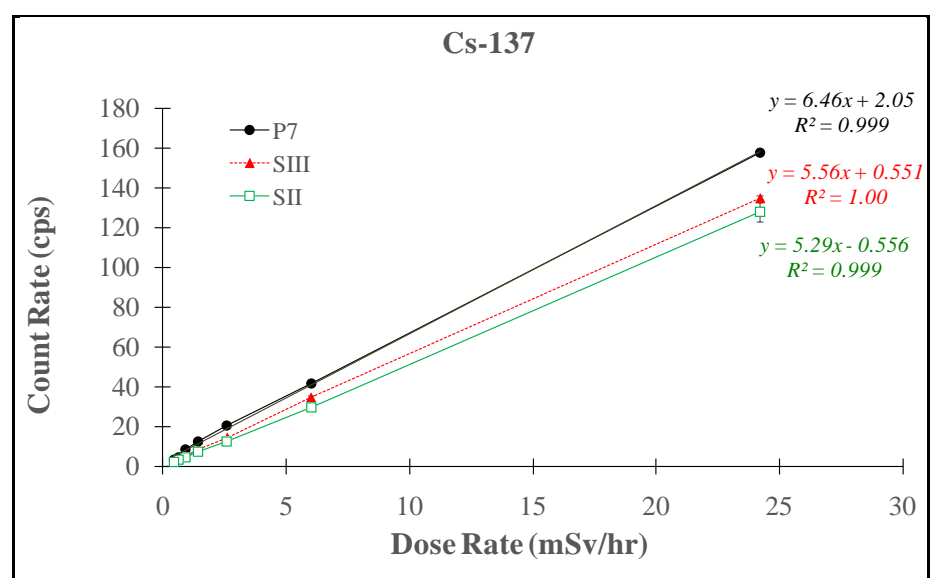

Figure 4. Gamma count rates vs the dose rates from Cs-137 source.

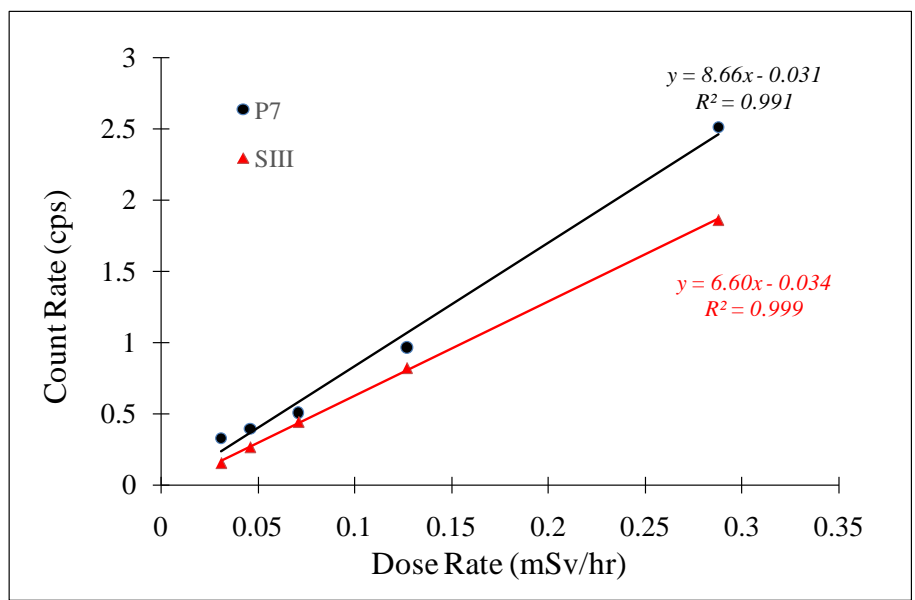

Figure 5. Gamma count rates vs the dose rates from low activity Co-60 source.

screens. The application software allowed user to perform counting from the saved video file or near real-time during measurement. User could input calibration equation to convert the counting rate to dose rate. Parts of the Application software menus are illustrated in Figure 7 and Figure 8. The application software has setting 


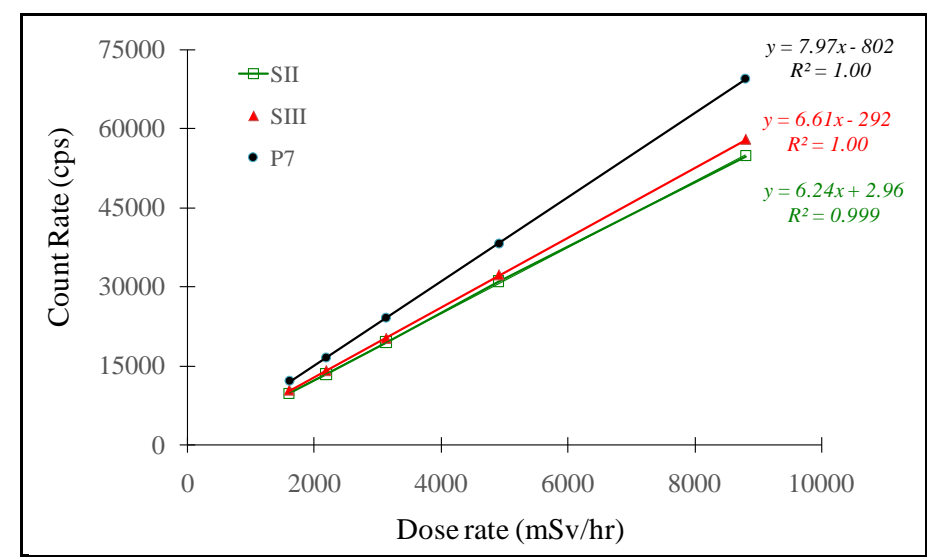

Figure 6. Gamma count rates vs the dose rates from high activity Co-60 source.

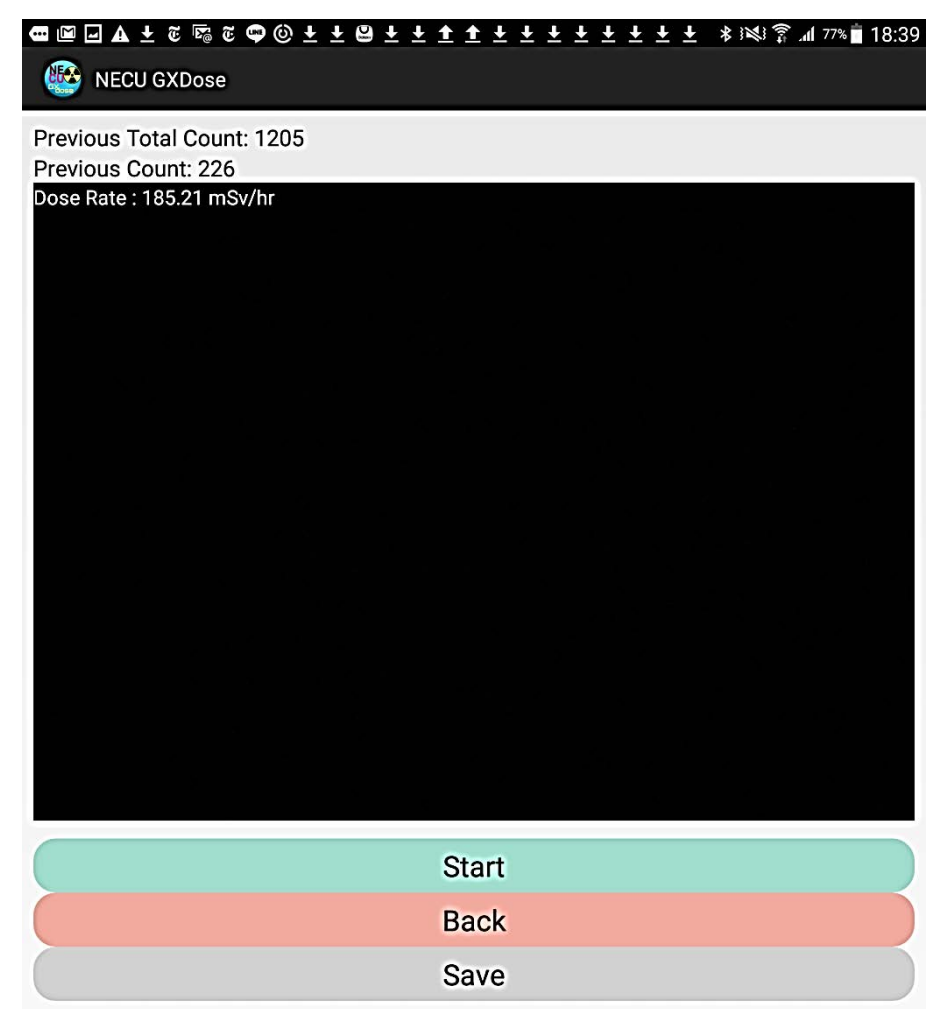

Figure 7. Set-up menus of the application software run on an Android smartphone.

parameters for threshold level to cut-off noisy signals, minimum and maximum pixel size to be counted, counting time and counting cycle. The number of counts is displayed near real-time but the dose rate will be displayed after the preset counting cycle is completed. Dose rate measurements by using the smartphones with the application software were conducted at the calibration facility of the Office of Atoms for Peace (OAP) in comparison with the standard ionization chamber. The results were very satisfactory as illustrated in Table 3.

\section{Results and Discussion}

The video images showed flashing of bright spots randomly appeared on the screen. It could be clearly noticed that number of the bright spots increased with the dose rate. It was found that the count rates increased linearly 


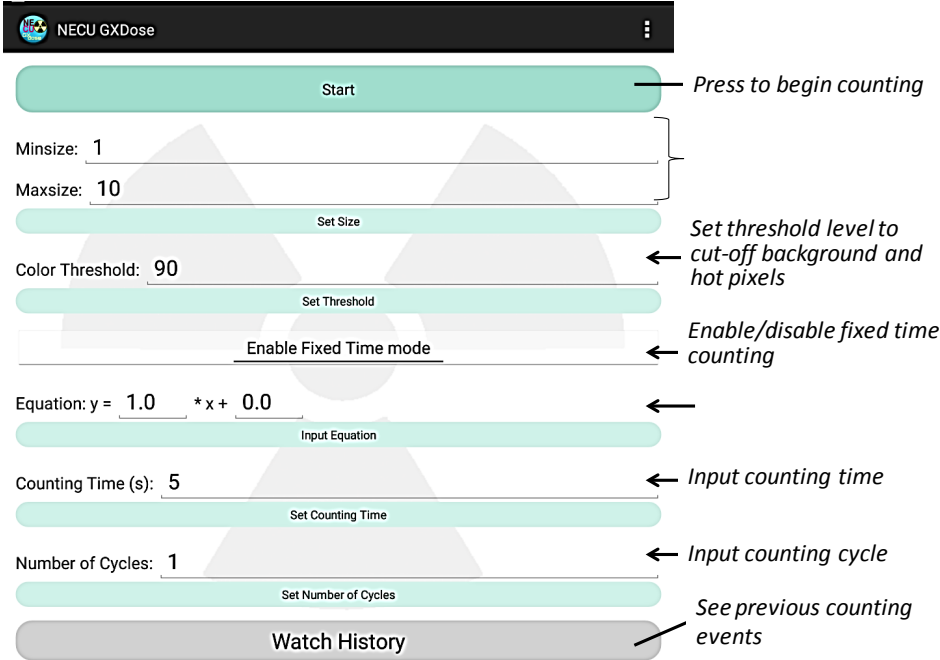

Figure 8. Brief description of the application software run on an Android smartphone.

Table 3. Comparison the dose rates from ${ }^{137}$ Cs source read by Huawei Ascend P7 in comparison with the known values and the values obtained from a standard ionization chamber.

\begin{tabular}{ccccc}
\hline \multirow{2}{*}{$\begin{array}{c}\text { Actual dose rate } \\
(\mathrm{mSv} / \mathrm{hr})\end{array}$} & \multicolumn{2}{c}{ Read by survey meter } & \multicolumn{2}{c}{ Read by Huawei Ascend P7 } \\
\cline { 2 - 5 } & Dose rate $(\mathrm{mSv} / \mathrm{hr})$ & Discrepancy & Dose rate $(\mathrm{mSv} / \mathrm{hr})$ & Discrepancy \\
\hline 0.545 & 0.547 & +0.002 & 0.525 & -0.020 \\
0.346 & 0.350 & +0.004 & 0.352 & +0.006 \\
0.239 & 0.241 & +0.002 & 0.235 & -0.004 \\
0.176 & 0.176 & 0.000 & 0.177 & +0.001 \\
0.133 & 0.132 & -0.001 & 0.132 & -0.001 \\
\hline
\end{tabular}

with increase of the dose rates in wide dose rate ranges for all tested smartphones as illustrated in Figures 3-6. Even at very high dose rate from ${ }^{60} \mathrm{Co}$ source. Sensitivities of the smartphones were found to be quite different. Among the four tested smartphones, Huawei Ascend P7 had highest sensitivity, the second was Samsung Galaxy S III, the third was Samsung Galaxy S II and the least sensitive was Samsung Galaxy GT-S5570 mini. Therefore, each smartphone needs to be calibrated to obtain its own calibration equation as normally do with radiation survey meters and dosimeter. It should be kept in mind that, like all survey meters and dosimeters, if they are calibrated with ${ }^{137} \mathrm{Cs}$ they can only give correct dose rate for ${ }^{137} \mathrm{Cs}$ gamma-rays. Correction factors are requires for other gamma-ray energies. This is because all detectors are energy dependent.

\section{Conclusion}

The tested results clearly confirmed that CMOS chip of smartphone's digital camera was sensitive to gammarays. When the camera lens was covered with black adhesive tape, interaction of gamma-rays with CMOS chip caused flashing bright spots on dark background which could be counted with appropriate software to obtain count rate as in radiation counters. Furthermore, the linear relationship between the count rate and the count rate in wide range has made conversion from count rate to dose rate simpler. However, other smartphones may be more sensitive than the ones tested in this research but they were not bought to test due to limited budget at this preliminary investigation. From our experience, the sensitivity depends very much on size of the CMOS chip not the image resolution or total number of image pixels. Our brief test of some of newly released smartphones with 13 MP (megapixels) camera showed that they were much less sensitive to gamma-rays than Huawei Ascend P7(13 MP) and Samsung Galaxy S III (8 MP). However, with the in-house developed Application software 
and appropriate calibration, any Android smartphones can be used to measure gamma-rays but having different sensitivities. The smartphones are therefore useful for radiation workers particularly for those who work with high activity radiation sources like industrial radiographers, radiologists, radiation technicians, gamma irradiation plant workers and others. For example, industrial radiography normally uses ${ }^{192} \mathrm{Ir}$ with activity of about 100 curies (Ci). From Table 2, $1 \mathrm{Ci}^{192} \mathrm{Ir}$ gives $5 \mathrm{mSv} / \mathrm{hr}$ at 1 meter. Thus $100 \mathrm{Ci}$ will give the dose rate at 1 meter as high as $500 \mathrm{mSv} / \mathrm{hr}$ or $20 \mathrm{mSv} / \mathrm{hr}$ at 5 meters without shielding materials. If the worker stays at 5 meters for an hour, he will receive a total dose of $20 \mathrm{mSv}$ which is equivalent to annual dose limit for radiation workers. Radiation workers with smartphones and the Application software can, therefore, have their own radiation measuring devices for better radiation safety and control. In-house developed Application software is now being improved and the one for iPhone is also under development.

\section{Acknowledgements}

We are grateful to the Department of Medical Sciences and the Office of Atoms for Peace (OAP) for allowing us to use their dose calibration facilities and materials. We would like to express our deepest thanks to Assistant Professor Attaporn Pattarasumunt for valuable discussions and suggestion as well as to Mr. Chalaempong Polee and Miss Nuatawan Thamrongsiripak for their suggestions and assistance during experiments. Thanks are also extended to Miss Tayida Tapjinda for her valuable assistance in developing the in-house Android application software.

\section{References}

[1] Akaranate, A., Chankow, N. and Pattarasumunt, A. (2013) Fast and Low Cost X-Ray Fluoroscopy Displayed on a 3D Monitor. Open Journal of Applied Sciences, 3, 308-311. http://dx.doi.org/10.4236/ojapps.2013.34039

[2] Iamsamang, K. and Chankow, N. (2013) Low Cost Gamma-Ray Fluoroscopic System for Se-75 and Ir-192 Industrial Radiography Sources. NDT Spectra (Official Journal of Malaysian Society for Non-Destructive Testing), No. 7, 209216.

[3] Gumiela, M. and Kozik, R. (2012) Studies of the Applicability of CMOS and CCD Sensors for Detection, Dosimetry and Imaging of Alpha, Beta, Gamma, X-Ray and Proton Beam Spots. Studies of the Applicability of CMOS and CCD Sensors for Detection, Dosimetry and Imaging of Alpha, Beta, Gamma, X-Ray and Proton Beam Spots, 2012.

[4] Cogliati, J.J., Derr, K.W. and Wharton, J. (2014) Using CMOS Sensors in a Cellphone for Gamma Detection and Classification. Using CMOS Sensors in a Cellphone for Gamma Detection and Classification, 26.

[5] Lederer, C.M., Hollander, J.M. and Perlman, I. (1967) Table of Isotopes. 6 Edition, John Wiley \& Sons, New York.

[6] (1992) International Atomic Energy Agency, Industrial radiography. IAEA, Vienna.

[7] Studio, A.D. (2008) Aoaophoto Video to Picture Converter 4.0.

[8] Ferreira, T. and Rasband, W. (2012) ImageJ User Guide: IJ1.46r. http://imagej.nih.gov/ij/docs/guide 